DFTT 83/95

hep-ph/9512353

\title{
Neutrino oscillations with three-generation mixings and mass hierarchy̋̋
}

\author{
S.M. Bilenky ${ }^{\mathrm{a}}$, A. Bottino ${ }^{\mathrm{b}}$, C. Giunti ${ }^{\mathrm{b}}$ and C. W. Kim ${ }^{\mathrm{c}}$ \\ a Joint Institute for Nuclear Research, Dubna, Russia. \\ bINFN and Dipartimento di Fisica Teorica, Università di Torino, \\ Via P. Giuria 1, 10125 Torino, Italy. \\ 'Department of Physics and Astronomy, The Johns Hopkins University, \\ Baltimore, Maryland 21218, USA.
}

\begin{abstract}
We have analyzed the results of reactor and accelerator neutrino oscillation experiments in a model with mixing of three neutrino fields and a neutrino mass hierarchy. It is shown that $\nu_{\mu} \leftrightarrows \nu_{e}$ oscillations with $0.6 \leq \Delta m^{2} \leq 100 \mathrm{eV}^{2}$ and amplitude larger than $2 \times 10^{-3}$ are not compatible with the existing limits on neutrino oscillations if the non-diagonal elements of the mixing matrix are small. Thus, if the excess of positron events recently observed in the LSND experiment is due to $\bar{\nu}_{\mu} \rightarrow \bar{\nu}_{e}$ oscillations, the mixing in the lepton sector is basically different from the CKM mixing of quarks.
\end{abstract}

*Talk presented by C. Giunti at TAUP 95, Toledo (Spain), September 17-21, 1995 
The LSND collaboration has recently reported [1] to have found some positive indications in favor of $\bar{\nu}_{\mu} \rightarrow \bar{\nu}_{e}$ transitions. We will discuss here the implications of this indication in favor of neutrino oscillations in the framework of a model with mixing among three massive neutrino fields and a mass hierarchy that can allow to accommodate the solar neutrino data [2].

The probability of $\nu_{\alpha} \rightarrow \nu_{\beta}$ transitions can be written in the following form:

$$
P_{\nu_{\alpha} \rightarrow \nu_{\beta}}=\left|\sum_{k=2}^{3} U_{\beta k}\left(\mathrm{e}^{-i \frac{\Delta m_{k 1}^{2} L}{2 p}}-1\right) U_{\alpha k}^{*}+\delta_{\beta \alpha}\right|^{2}
$$

Here $L$ is the distance between the neutrino source and detector, $\Delta m_{k 1}^{2} \equiv m_{k}^{2}-m_{1}^{2}$ and $p$ is the neutrino momentum. We will assume that $m_{1} \ll m_{2} \ll m_{3}$ and that $\Delta m_{21}^{2}$ is relevant for the possible suppression of the solar $\nu_{e}$ flux. In this case, for experiments with terrestrial neutrinos $\Delta m_{21}^{2} L / 2 p \ll 1$. The probability of $\nu_{\alpha} \rightarrow \nu_{\beta}$ transitions and the probability of $\nu_{\alpha}$ to survive are given by (see Ref.[3])

$$
\begin{aligned}
& P_{\nu_{\alpha} \rightarrow \nu_{\beta}}=\frac{A_{\nu_{\alpha} ; \nu_{\beta}}}{2}\left(1-\cos \frac{\Delta m^{2} L}{2 p}\right), \quad \alpha \neq \beta, \\
& P_{\nu_{\alpha} \rightarrow \nu_{\alpha}}=1-\frac{B_{\nu_{\alpha} ; \nu_{\alpha}}}{2}\left(1-\cos \frac{\Delta m^{2} L}{2 p}\right),
\end{aligned}
$$

with $\alpha, \beta=e, \mu, \tau$ and $\Delta m^{2} \equiv \Delta m_{31}^{2}$. Here

$$
\begin{aligned}
& A_{\nu_{\alpha} ; \nu_{\beta}}=4\left|U_{\alpha 3}\right|^{2}\left|U_{\beta 3}\right|^{2} \\
& B_{\nu_{\alpha} ; \nu_{\alpha}}=\sum_{\beta \neq \alpha} A_{\nu_{\alpha} ; \nu_{\beta}}=4\left|U_{\alpha 3}\right|^{2}\left(1-\left|U_{\alpha 3}\right|^{2}\right)
\end{aligned}
$$

are the amplitudes of oscillations. Let us emphasize that in the model under consideration all the three oscillation channels $\left(\nu_{\mu} \leftrightarrows \nu_{e}, \nu_{\mu} \leftrightarrows \nu_{\tau}, \nu_{e} \leftrightarrows \nu_{\tau}\right)$ are open. The oscillations in all channels are characterized by the same oscillation length $L_{\mathrm{osc}}=4 \pi p / \Delta m^{2}$. The oscillation amplitudes (11) and (2) are determined by two mixing parameters, which can be chosen to be $\left|U_{e 3}\right|^{2}$ and $\left|U_{\mu 3}\right|^{2}$. From the unitarity of the mixing matrix we have $\left|U_{\tau 3}\right|^{2}=1-\left|U_{e 3}\right|^{2}-\left|U_{\mu 3}\right|^{2}$.

In order to understand the implications of the LSND positive signal in the framework of this model, we have analyzed the negative results of all the other neutrino oscillation experiments with terrestrial neutrinos. We have used the results of the Bugey reactor experiment [4] on the search for $\bar{\nu}_{e}$ disappearance, of the CDHS and CCFR accelerator experiments [5] on the search for $\nu_{\mu}$ disappearance, of the BNL E776 and KARMEN accelerator experiments [6] on the search for $\nu_{\mu} \rightarrow \nu_{e}$ transitions and of the FNAL E531 accelerator experiment [7] on the search for $\nu_{\mu} \rightarrow \nu_{\tau}$ transitions. For $\Delta m^{2}$ we have considered the interval $10^{-1} \mathrm{eV}^{2} \leq \Delta \mathrm{m}^{2} \leq 10^{2} \mathrm{eV}^{2}$, that covers the LSND-allowed region [1]. From the results of reactor and accelerator disappearance experiments, at fixed values of $\Delta m^{2}$, the allowed values of the amplitudes $B_{\nu_{e} ; \nu_{e}}$ and $B_{\nu_{\mu} ; \nu_{\mu}}$ are constrained by

$$
B_{\nu_{e} ; \nu_{e}} \leq B_{\nu_{e} ; \nu_{e}}^{0} \quad \text { and } \quad B_{\nu_{\mu} ; \nu_{\mu}} \leq B_{\nu_{\mu} ; \nu_{\mu}}^{0} .
$$


From Eqs.(2) and (3) it follows that the parameters $\left|U_{e 3}\right|^{2}$ and $\left|U_{\mu 3}\right|^{2}$ must be either small or large (close to one). For example, at $\Delta m^{2}=6 \mathrm{eV}^{2}$ we have $\left|U_{e 3}\right|^{2} \leq 0.04$ or $\left|U_{e 3}\right|^{2} \geq 0.96$ and $\left|U_{\mu 3}\right|^{2} \leq 0.02$ or $\left|U_{\mu 3}\right|^{2} \geq 0.98$.

Since from the unitarity of the mixing matrix the parameters $\left|U_{e 3}\right|^{2}$ and $\left|U_{\mu 3}\right|^{2}$ cannot be both close to one, in the following we will consider the three possible regions for these parameters.

I. $\left|U_{e 3}\right|^{2}$ and $\left|U_{\mu 3}\right|^{2}$ are both small.

In this region $\left|U_{\tau 3}\right|^{2}$ is close to one and from the results of the FNAL E531 experiment on the search for $\nu_{\mu} \rightarrow \nu_{\tau}$ transitions we obtain rather strong limitations on the value of $\left|U_{\mu 3}\right|^{2}$ and, consequently, on the $\nu_{\mu} \rightarrow \nu_{e}$ oscillation amplitude $A_{\nu_{\mu} ; \nu_{e}}$. The results of our calculations are presented in Fig. 1 in the form of exclusion regions in the $A_{\nu_{\mu} ; \nu_{e}}-\Delta m^{2}$ plane. The region allowed by the LSND experiment is represented as the shadowed region limited by the two solid curves. The curve passing through the filled circles is the boundary of the excluded region that was obtained from the results of the Bugey, CDHS and CCFR disappearance experiments. The curve passing through the open circles was obtained by combining the results of the Bugey experiment and the FNAL E531 experiment on the search for $\nu_{\mu} \rightarrow \nu_{\tau}$ transitions. The dashed line reproduces the results of the Bugey disappearance experiment (we took into account the fact that $A_{\nu_{\mu} ; \nu_{e}} \leq B_{\nu_{e} ; \nu_{e}}$ ). The dotted and dash-dotted lines reproduce, respectively, the results of the BNL E776 and KARMEN experiments on the search for $\nu_{\mu} \rightarrow \nu_{e}$ transitions.

From Fig.1 it can be seen that, in the framework of the model under consideration, the results of $\nu_{e}$ and $\nu_{\mu}$ disappearance experiments together with the results of the FNAL E531 $\nu_{\mu} \rightarrow \nu_{\tau}$ appearance experiment provide more severe restrictions on $A_{\nu_{\mu} ; \nu_{e}}$ than the results of direct $\nu_{\mu} \rightarrow \nu_{e}$ appearance experiments. Furthermore, practically all the LSNDallowed region is inside the region which is forbidden by the results of the other reactor and accelerator experiments. Therefore, if the results of the LSND experiment will be confirmed, it will mean that the parameters $\left|U_{e 3}\right|^{2}$ and $\left|U_{\mu 3}\right|^{2}$ cannot be both small.

II. $\left|U_{e 3}\right|^{2}$ is large and $\left|U_{\mu 3}\right|^{2}$ is small.

This region is forbidden by the solar neutrino data. In fact, in the case of a neutrino mass hierarchy, the survival probability of the solar neutrinos is given by [9]

$$
P_{\nu_{e} \rightarrow \nu_{e}}=\left(1-\left|U_{e 3}\right|^{2}\right)^{2} P_{\nu_{e} \rightarrow \nu_{e}}^{(1,2)}+\left|U_{e 3}\right|^{4},
$$

where $P_{\nu_{e} \rightarrow \nu_{e}}^{(1,2)}$ is the survival probability due to the mixing between the first and the second generations. In the interval of $\Delta m^{2}$ under consideration, we have $P_{\nu_{e} \rightarrow \nu_{e}} \geq 0.92$ for all values of the neutrino energy. With such a high lower bound for the $\nu_{e}$ survival probability it is not possible to explain the results of the solar neutrino experiments [8].

III. $\left|U_{e 3}\right|^{2}$ is small and $\left|U_{\mu 3}\right|^{2}$ is large.

In this region, in the linear approximation over the small parameters $\left|U_{e 3}\right|^{2}$ and $(1-$ $\left|U_{\mu 3}\right|^{2}$ ) we have

$$
\begin{aligned}
& A_{\nu_{\mu} ; \nu_{e}} \simeq 4\left|U_{e 3}\right|^{2}, \\
& A_{\nu_{\mu} ; \nu_{\tau}} \simeq 4\left(1-\left|U_{\mu 3}\right|^{2}-\left|U_{e 3}\right|^{2}\right), \\
& A_{\nu_{e} ; \nu_{\tau}} \simeq 0 .
\end{aligned}
$$


From Eq.(6) and the unitarity relations (2) it follows that

$$
\begin{aligned}
& A_{\nu_{\mu} ; \nu_{e}} \simeq B_{\nu_{e} ; \nu_{e}}, \\
& A_{\nu_{\mu} ; \nu_{\tau}} \simeq B_{\nu_{\mu} ; \nu_{\mu}}-B_{\nu_{e} ; \nu_{e}} .
\end{aligned}
$$

In Ref.[2] we have shown that in region III the LSND positive signal is compatible with the negative results of all the other experiments on the search for neutrino oscillations. Thus, a confirmation of the LSND result will constrain the parameters $\left|U_{e 3}\right|^{2}$ and $\left|U_{\mu 3}\right|^{2}$ to lie in region III. In this case $\nu_{\mu} \rightarrow \nu_{\tau}$ oscillations that are searched for in the CHORUS and NOMAD experiments [10] are allowed and $\nu_{e} \rightarrow \nu_{\tau}$ oscillations are strongly suppressed (for example, $A_{\nu_{e} ; \nu_{\tau}} \lesssim 3 \times 10^{-5}$ at $\Delta m^{2}=6 \mathrm{eV}^{2}$ ). If a positive signal will be found in both $\nu_{\mu} \rightarrow \nu_{e}$ and $\nu_{\mu} \rightarrow \nu_{\tau}$ channels, using Eqs.(1) and (5) it will be possible to determine the values of the mixing parameters $\left|U_{\mu 3}\right|^{2}$ and $\left|U_{e 3}\right|^{2}$. In this case, the validity of the model under discussion can be tested by checking the presence of only one relevant mass scale $\Delta m^{2}$ for all the oscillation channels.

In conclusion, we have considered a model with mixing of three neutrino fields and a neutrino mass hierarchy that can accommodate the results of the solar neutrino experiments. After the calibration of the GALLEX detector with a radioactive source 11 the indications in favor of neutrino oscillations coming from solar neutrino experiments have become more significant. We think that the model that we have considered is the simplest and most realistic model of neutrino mixing. It seems very appropriate to analyze in the framework of this model all the data from the existing experiments on the search for neutrino oscillations and to infer predictions for the results of future experiments (see Ref. [12]). Our analysis has shown that a confirmation of the LSND positive result for $\nu_{\mu} \rightarrow \nu_{e}$ oscillations would imply that $\left|U_{e 3}\right|^{2}$ is small and $\left|U_{\mu 3}\right|^{2}$ is large in the scheme under discussion. This result would mean that neutrino mixing is basically different from quark mixing: there is no natural hierarchy of coupling in the lepton sector and $\nu_{\mu}$ is the "heaviest" neutrino.

\section{References}

[1] C. Athanassopoulos et al., Phys. Rev. Lett. 75 (1995) 2650.

[2] S.M. Bilenky et al., Phys. Lett. B 356 (1995) 273.

[3] A. De Rujula et al., Nucl. Phys. B 168 (1980) 54; V. Barger and K. Whisnant, Phys. Lett. B 209 (1988) 365; S.M. Bilenky et al., ibid 276 (1992) 223.

[4] B. Achkar et al., Nucl. Phys. B 434 (1995) 503.

[5] F. Dydak et al., Phys. Lett. B 134 (1984) 281; I.E. Stockdale et al., Phys. Rev. Lett. 52 (1984) 1384.

[6] L. Borodovsky et al., Phys. Rev. Lett. 68 (1992) 274; B. Armbruster et al., Nucl. Phys. B (Proc. Suppl.) 38 (1995) 235. 
[7] N. Ushida Phys. Rev. Lett. 57 (1986) 2897.

[8] B.T. Cleveland et al., Nucl. Phys. B (Proc. Suppl.) 38 (1995) 47; K. S. Hirata et al., Phys. Rev. D 44 (1991) 2241; GALLEX Coll., Phys. Lett. B 357 (1995) 237; V.N. Gavrin, Talk presented at TAUP 95 Toledo (Spain), Sept. 1995.

[9] T.K. Kuo and J. Pantaleone, Phys. Rev. Lett. 57 (1986) 1805; X. Shi and D.N. Schramm, Phys. Lett. B 283 (1992) 305.

[10] K. Winter, Nucl. Phys. B (Proc. Suppl.) 38 (1995) 211; L. Di Lella, Nucl. Phys. B (Proc. Suppl.) 31 (1993) 319.

[11] GALLEX Coll., Phys. Lett. B 342 (1995) 440.

[12] G.L. Fogli, E. Lisi and D. Montanino, Phys. Rev. D 49 (1994) 3626; CERN-TH-749194. 


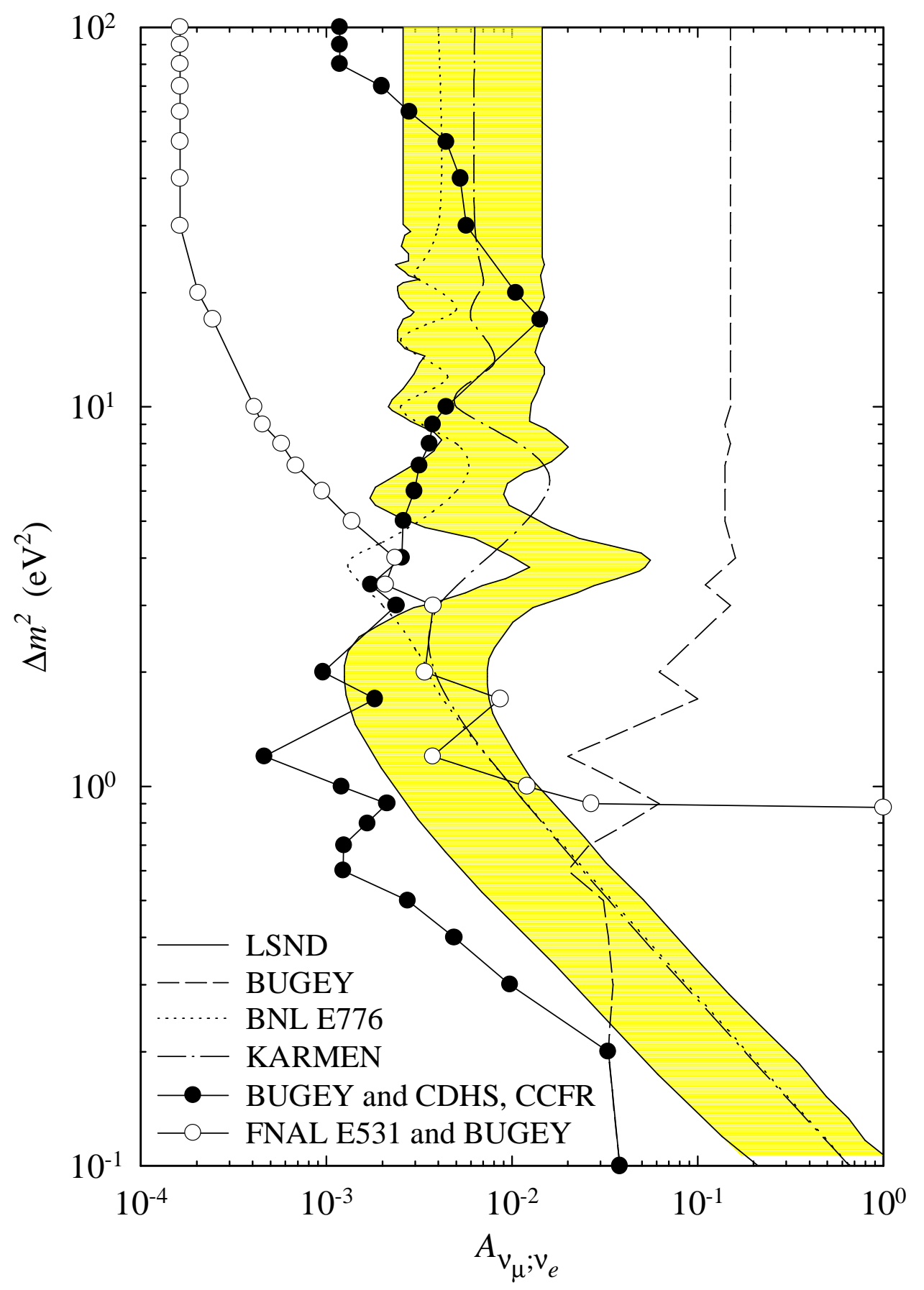

Figure 1 